\title{
A Case of Post-Traumatic Stress Disorder
}

\author{
Alberta Shkembi*, Elda Skenderi and Feride Imeraj \\ Department of Pediatrics, University Hospital Center "Mother Teresa", Albania
}

*Corresponding author: Alberta Shkembi, Department of Pediatrics, University Hospital Center "Mother Teresa", Tirana, Albania.

Received Date: July 03, 2020

Published Date: July 21, 2020

\begin{abstract}
s
This article presents a case study of posttraumatic stress disorder. Patient also manifest somatic symptoms, these symptoms affect her daily functionality. The purpose of this article is to identify the symptoms of post-traumatic stress. The working hypothesis are: The effects of traumatic events that have occurred are related to the psychological sensitivity that the patient exhibits. Traumatic events can trigger somatic sensations such as headaches and high temperature. The goal of treatment is to reduce negative thoughts by coping with negative experience.
\end{abstract}

Key words: Post-traumatic stress; Somatic symptoms; Negative experience

\section{Introduction}

Past traumatic situations can affect the feeling of security and perspective on life. Traumatic events of childhood and somatization. Common somatic complaints of schoolchildren such as headache, stomachache are associated with psychosocial variables of anxiety and depression, childhood trauma and school distress. Somatic symptoms in children with somatization disorder become the focus of their attention and often interfere with school, home life, and relationships with peers Bernard. The somatization disorder typically comes after an experience of a psychological stressor. Somatization often occurs in response to psychological stress, and usually persists even after the acute stressor has gone away, leading to a situation where both, the child and the family, believe that the medical problem has not yet been found. This leads to a continuous searching for the correct medical diagnosis. Concerns, conscious or unconscious, can lead to somatic symptoms that vary in severity and can affect any organ Beck.

In the past, emotional and physical events were considered separate, considering the body and mind unaffected by each other. However, today this view has changed. Now it is well known that these two factors constantly influence each other. Psychological trauma affects biological processes and vice versa. Psychological trauma can damage the endocrine system. Also, exposure to extreme stress triggers the "fight or go" response, which activates the sympathetic system. This response increases the level of cortisol in the CNS, which allows the individual to take survival actions, but at extreme levels affects brain development. In children, high cortisol levels can disorder cell differentiation, cell migration, or other CNS functions. Trauma also affects the limbic system of the brain (responsible foremotions, memory, affection, regulation of stimulation), the neocortex (perception of oneself and the world) and other functions in the CNS. Traumatic experiences are stored in the child's mind / body and pose a constant threat to his or her well-being. The literature on the traumatic effects of trauma focuses on post-traumatic stress. Somatization is more likely to occur in children and adolescents who are abused or have suffered all types of trauma. Childhood trauma is a risk factor for somatization, Rief Hiller W, Hesueser (1997) compared 54 patients with somatization disorder and 50 with mood disorders and measured childhood trauma. They found that individuals with somatic disorder had childhood traumas such as physical abuse, sexual abuse, or violence of various forms more than those suffering from depression. Child- 
hood traumas often lead to somatization symptoms which are related to a number of symptoms that do not have a medical explanation including chronic pain, headache, gastrointestinal symptoms, and musculoskeletal symptoms.

Although most studies have examined only physical and sexual abuse, recent studies have shown that emotional abuse and neglect are also associated with somatization. Children can also experience a range of traumatic situations at school that can trigger somatization. Bullying, school violence, exposure to a traumatic event on the school premises, and high expectations for the child's achievements have been estimated to lead to somatization. Stress is defined as a causative factor. The most common forms of stress consist of the pressure exerted on the child to learn Charls.

\section{Patient Identification}

E.L is the youngest child in the family. The patient lives with her mother and her 17-year-old brother. She is presented to the Pediatric Service due to a long consistent temperature of two weeks. During her stay in hospital, it was never concluded that the girl had temperature. During the evaluation session with her, it is noticed that the patient shows many fears such as: Fear of being stealed, fear that her mother may lose her life from the current. She is extremely preoccupied with being different from her friends. "I'm not like them " - she says, -", that's why I don't socialize with none of them "What she mostly wants to change is for others to love her, also, she adds, that there are constant deviations from activities outside the home. The girl's mother reports that mornings are frequent when E.L wakes up and tells about the sad dreams she has had during the night, with wild animals that she does not know, and does not know how to explain their appearance. The mother also adds that the girl has many difficulties with sleep, sometimes she has more difficulty sleeping and other times she falls asleep at night. The main concern of the patient is the feeling of being sick. This condition disrupts her daily functioning and she is continuously thinking that something bad will happen to her family in the future

\section{Precipitants and Activating Situations}

The patient's mother is extremely concerned about the patient's lack of tolerance to candle lighting in cases where there is a lack of electricity in the house. The mother claims that E.L bursts into tears and has a nervous breakdown if we light a candle. We have to turn it off so that E.L can calm down, the mother continues. Otherwise she starts complaining of headaches and high temperature. E. $\mathrm{L}$ is a regular patient in the pediatric service and her visits occur almost once every two months. Her complaints are: high temperature and unbearable headaches. Specific examinations of these complaints indicate a lack of any possible organic problems.

\section{Origins/Early Experiences}

E.L was present at a serious event that took place in her family. The girl's father was deliberately burned in the presence of her, her brother and their mother. The incident happened when E.L was 13 and a half years old. It is important to note that the patient's father has been a chronic alcoholic, has exercised systematic violence against his wife and children, and there has also been an episode of partial burning of the house when Enia was 9 years old.

\section{Treatment Plan}

\section{Goals}

The goal of treatment is to reduce negative thoughts by coping with negative experience.

\section{Interventions}

- Monitoring emotions and Edb in context - The Arc of Emotions

- $\quad$ Thoughts- Emotions- Sensation - Behavior Monitoring

- $\quad$ Thought Challening Chart (Barlow, 2011)

8. Conclusion

- $\quad$ Predicted Obstacles to Treatment

- Lots of family problems

- Feeling bias that she is taking psychological service

- Emotional health of her mother

\section{Acknowledgement}

None.

\section{Conflict of Interest}

No conflict of interest.

\section{References}

1. Beck J (2006) Trajectories, antecedents, and outcomes of childhood somatization. Refered to: etd.library.pitt.edu

2. Bernard WK Lau, Wilson WC TSE (2000) Psychological effects of Physical illness and hospitalization on the child and the family.

3. Charles C, Engel jr (2004) somatization and multiple idiopathic physical symptoms: relationship to traumaticevents and posttraumatic stress disorder. Washington, DC, US: American Psychological Association.

4. Rief W, Hiller W, Hesueser J (1997) Soms- The screening for Somatoform Symptoms) Bern, Switzerland: Huber- Verlag.

5. David H Barlow, Tod J Farchione, Christopher P Fairholme, Kristen K Ellard, Christina L Boisseau, et al. (2011) Unifield Protocol for Transdiagonistic Treatment of Emotional Disorders . OXFORD University Press. 